\title{
Singleton and Twin Fetal Movements before 20 Weeks of Gestation
}

\author{
${ }^{1}$ Nobuhiro Mori, ${ }^{2}$ Kenji Kanenishi, ${ }^{3}$ Mohamed AM AboEllail, ${ }^{4}$ Emiko Nitta, ${ }^{5}$ Junko Noguchi, ${ }^{6}$ Genzo Marumo \\ ${ }^{7}$ Toshiyuki Hata
}

\begin{abstract}
Objective:To assess the change in the frequency of singleton and twin fetal movements with advancing gestation and compare the total number of fetal movements among singleton, active, and quiet twin fetuses at 12 to 19 weeks of gestation using four-dimensional (4D) ultrasound.
\end{abstract}

Materials and methods: The 4D ultrasound was used to examine fetal movements in 58 singleton and 48 normal twin fetuses at 12 to 19 weeks of gestation. The frequencies of eight fetal movements were assessed through 15-minute recordings. The correlation between the frequency of each fetal movement in singleton and twin fetuses and gestational age was analyzed. The total number of fetal movements among singleton, active, and quiet twin fetuses was compared at 12 to 13 and 14 to 19 weeks respectively.

Results: Frequencies of hand-to-face and leg movements were significantly increased with advancing gestation, whereas the frequencies of general movements significantly decreased at 12 to 19 weeks in singleton fetuses. Frequencies of body rotation and general movements were significantly decreased with advancing gestation, whereas the frequencies of mouthing movement significantly increased at 12 to 19 weeks in twin fetuses. The total number of fetal movements in singleton fetuses was significantly higher than that in quiet twins at 12 to 13 weeks of gestation, and there were significant differences in the total number of fetal movements between singleton fetuses and active or quiet twins at 14 to 19 weeks.

Conclusion: Our results suggest that the characteristics of fetal movements in singleton and twin fetuses are different before 20 weeks of gestation. However, the data and their interpretation in the present study should be taken with some degree of caution because of the small number of subjects studied. Further studies involving a larger sample size are needed to draw the hard conclusions regarding the difference in fetal movements between singleton and twin pregnancies before 20 weeks' gestation.

\footnotetext{
$1,3,4$ Assistant Professor, ${ }^{2}$ Associate Professor, ${ }^{5}$ Professor ${ }^{6}$ President, ${ }^{7}$ Professor and Chairman

1-4,7Department of Perinatology and Gynecology, Kagawa University Graduate School of Medicine, Miki, Kagawa, Japan

${ }^{5}$ Department of Nursing, Kagawa Prefectural College of Health Sciences, Takamatsu, Kagawa, Japan

${ }^{6}$ Department of Obstetrics and Gynecology, Marumo Ladies Clinic, Minato-ku, Tokyo, Japan
}

Corresponding Author: Toshiyuki Hata, Professor and Chairman, Department of Perinatology and Gynecology, Kagawa University Graduate School of Medicine, Miki, Kagawa, Japan Phone: +810878912174, e-mail: toshi28@med.kagawa-u.ac.jp
Keywords: Fetal movement, First half of pregnancy, Fourdimensional ultrasound, Singleton pregnancy, Twin pregnancy.

How to cite this article: Mori N, Kanenishi K, AboEllail MAM, Nitta E, Noguchi J, Marumo G, Hata T. Singleton and Twin Fetal Movements before 20 Weeks of Gestation. Donald School J Ultrasound Obstet Gynecol 2018;12(2):99-103.

\section{Source of support: Nil}

Conflict of interest: None

\section{INTRODUCTION}

With respect to singleton fetal movements assessed by $4 \mathrm{D}$ ultrasound at 7 to 14 weeks of gestation, the frequencies of general movements, stretching, isolated arm and leg movements, head retroflexion, head rotation, and head retroflexion were reported to significantly increase with advancing gestation, whereas the frequency of the startle movement was constant during this period. ${ }^{1}$ However, a significant difference was reported only in the frequency of mouthing movement assessed by $4 \mathrm{D}$ ultrasound between 14 to 16 and 17 to 19 weeks' gestation, with no significant differences in the other seven movements (head anteflexion, head retroflexion, body rotation, hand-to-face movement, general movement, isolated arm movement, and isolated leg movement) between 14 to 16 and 17 to 19 weeks' gestation. ${ }^{2}$ To the best of our knowledge, there has been no report on 4D ultrasound assessment of the change in the frequency of singleton and twin fetal movements with advancing gestation in the first half of pregnancy.

With respect to the twin fetal activity using 4D ultrasound between twin pairs at 11 to 14 weeks of gestation, there was a difference in the total counts of fetal movements (general body, isolated head, isolated arm, and isolated leg movements) between the active twins and their cotwins. ${ }^{3}$ Moreover, maternal reports on infants' temperament after birth and the more active twin in each pair were closely associated with prenatal intertwin differences in activity. ${ }^{3}$ Kurjak et $\mathrm{al}^{4}$ reported that twins showed less activity and different behavioral pattern than singletons in the third trimester of pregnancy. However, there has been no 4D ultrasound study on the assessment of singleton and twin fetal activity early in the second trimester of pregnancy. The current study was aimed to assess the change in the frequency of singleton and twin 
fetal movements with advancing gestation and compare the total number of fetal movements among singleton, active, and quiet twin fetuses at 12 to 19 weeks of gestation using $4 \mathrm{D}$ ultrasound.

\section{MATERIALS AND METHODS}

The design of the study, sample of patients, criteria for inclusion, and definitions of twin fetal movements were described in detail previously. ${ }^{5}$ Pregnant women who visited the outpatient clinic of Kagawa University Hospital for antenatal care were recruited. They included a total of 58 singleton and 25 twin [13 dichorionic diamniotic (DD) and 12 monochorionic diamniotic (MD) twins] pregnancies, and all subjects were the same with our previous study. ${ }^{5}$

Voluson E8 (GE Healthcare Japan, Tokyo, Japan) equipped with a curved array transabdominal transducer (1-4 MHz) was used in all cases. A 4-GB USB connected to the ultrasound machine was used to record all examinations, which were 15 minutes long. All examinations were conducted in the morning in a quiet, temperaturecontrolled room. A single examiner performed all examinations and data analysis (M.A.M.A.). The Kagawa University Graduate School of Medicine ethics committee approved this study, and all participants provided standardized written informed consent.

As those eligible for enrollment, 58 singleton fetuses and 48 fetuses of 25 pregnant women with twin pregnancies were enrolled. A total of two fetuses (one DD at 14 weeks and one MD at 18 weeks and 6 days) were excluded due to an unclear view of fetal movements. The eligible cases were then separated into two groups: Early gestation group (12-13 weeks) and later gestation group (14-19 weeks). The early group comprised 21 singleton fetuses and 7 twin pairs (2 MD and $5 \mathrm{DD}$ twin pregnancies). A total of 37 singleton fetuses and 16 twin

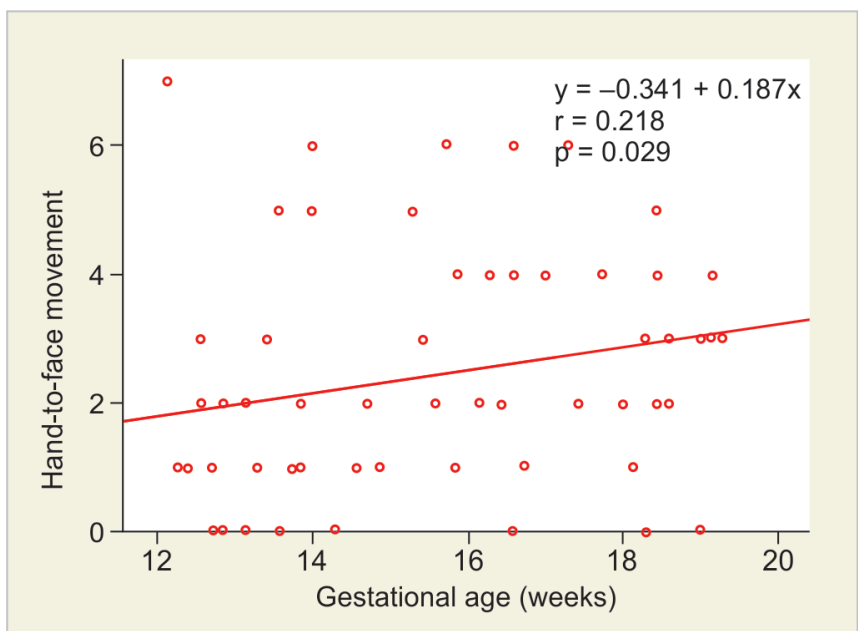

Graph 1: The frequency of singleton hand-to-face movement by gestational age pairs (9 MD and 7 DD twin pregnancies) comprised the later group.

As described in detail in our previous study, ${ }^{2}$ eight fetal movements (head anteflexion, head retroflexion, body rotation, hand-to-face movement, general movement, arm movement, leg movement, and mouthing) were assessed. An active twin fetus was defined as one showing a higher total frequency of the eight movements than the co-twin fetus in the same twin pregnancy. Good intra- and interclass correlation coefficients and intra- and interobserver agreements were also confirmed in that investigation. ${ }^{2}$ The frequencies of total movement are expressed as the median and range. The correlation between the gestational age and frequency of each of the eight fetal movements was assessed using Spearman's rank correlation coefficient. The total frequency of the eight movements among singleton, active, and quiet twin fetuses at 12 to 13 and 14 to 19 weeks' gestation was compared using the KruskalWallis one-way analysis of variance by ranks and multiple comparisons. Statistical Package for the Social Sciences statistical software, version 23 for windows (SPSS Inc., Chicago, Illinois, USA), was used for statistical analysis. A value of $\mathrm{p}<0.05$ was considered significant.

\section{RESULTS}

Frequencies of hand-to-face (Graph 1) and leg (Graph 2) movements in singleton fetuses were significantly increased with advancing gestation $(p=0.029$ and $p=0.005$ respectively), whereas the frequencies of general movements significantly decreased at 12 to 19 weeks $(p=0.045)$ (Graph 3). The frequencies of the other five fetal movements were constant at 12 to 19 weeks of gestation.

Frequencies of body rotation (Graph 4) and general (Graph 5) movements in twin fetuses were significantly decreased with advancing gestation ( $p=0.012$ and $p=0.0001$ respectively), whereas the frequencies of

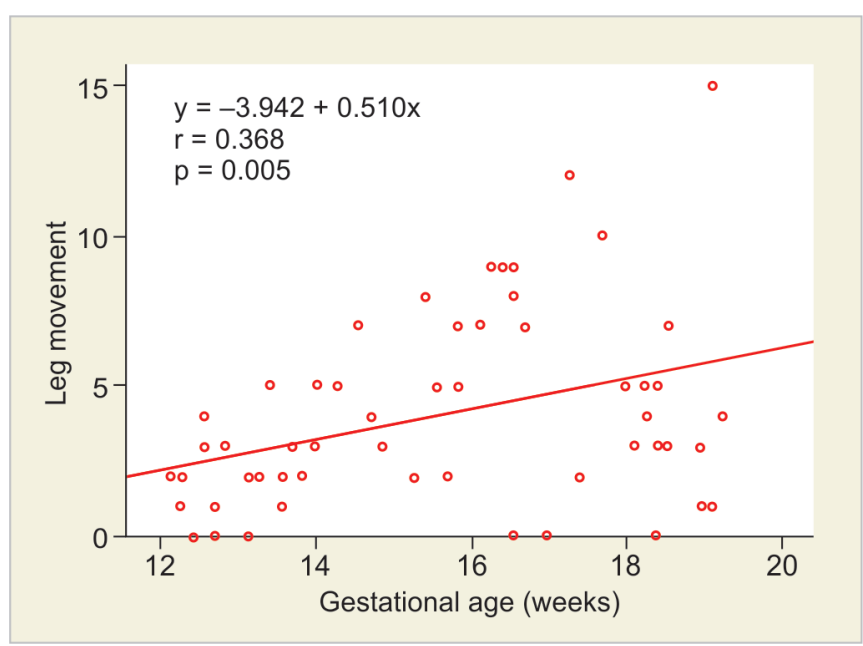

Graph 2: The frequency of singleton leg movement by gestational age 


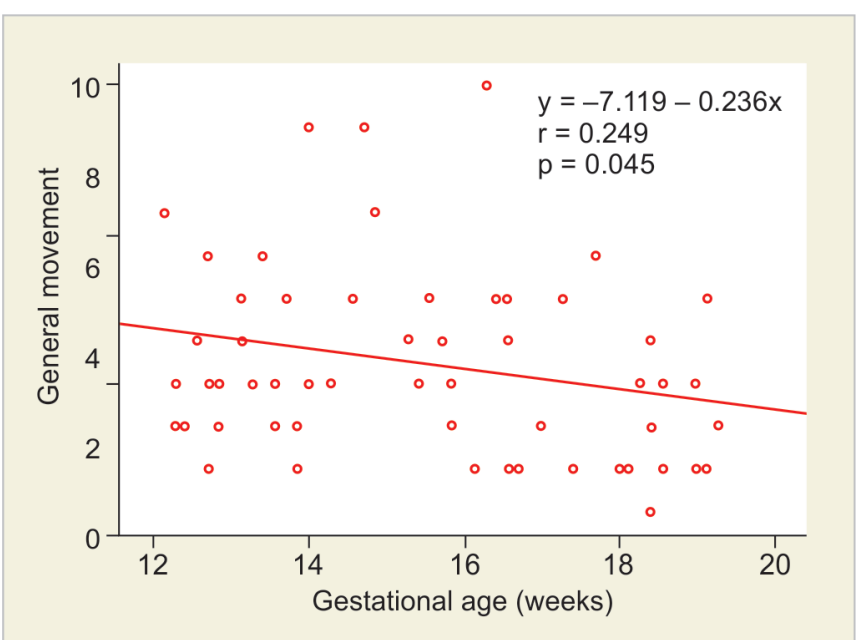

Graph 3: The frequency of singleton general movement by gestational age

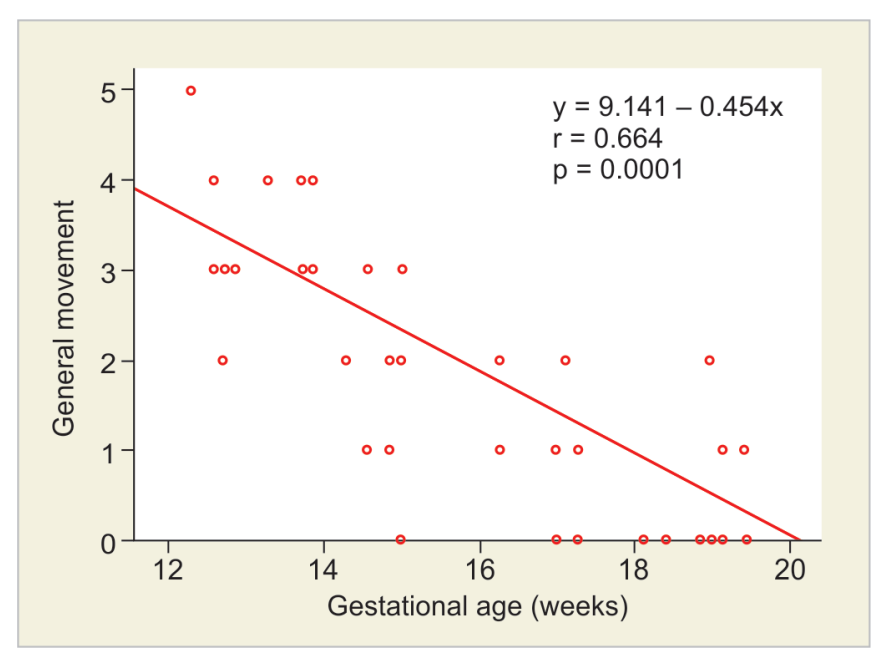

Graph 5: The frequency of twin general movement by gestational age

mouthing movement significantly increased at 12 to 19 weeks $(p=0.003)($ Graph 6). However, the frequencies of the other five fetal movements were constant at 12 to 19 weeks of gestation.

The total number of fetal movements in singleton fetuses (median, 16; 4-33) was significantly higher than that in quiet twins (median, 10;3-13) at 12 to 13 weeks of gestation, whereas there was no significant difference in the total number of fetal movements between singleton and active twin (median, 15; 7-21) fetuses (Graph 7). There were significant differences in the total number of fetal movements between singleton (median, 22; 2-52) and active (median, 9; 5-18) or quiet twin (median, 6; 2-16) fetuses at 14 to 19 weeks (Graph 7).

\section{DISCUSSION}

de Vries et $\mathrm{al}^{6}$ studied various fetal movements using conventional two-dimensional (2D) sonography in normal singleton pregnancies at 8 to 19 weeks of gestation. As

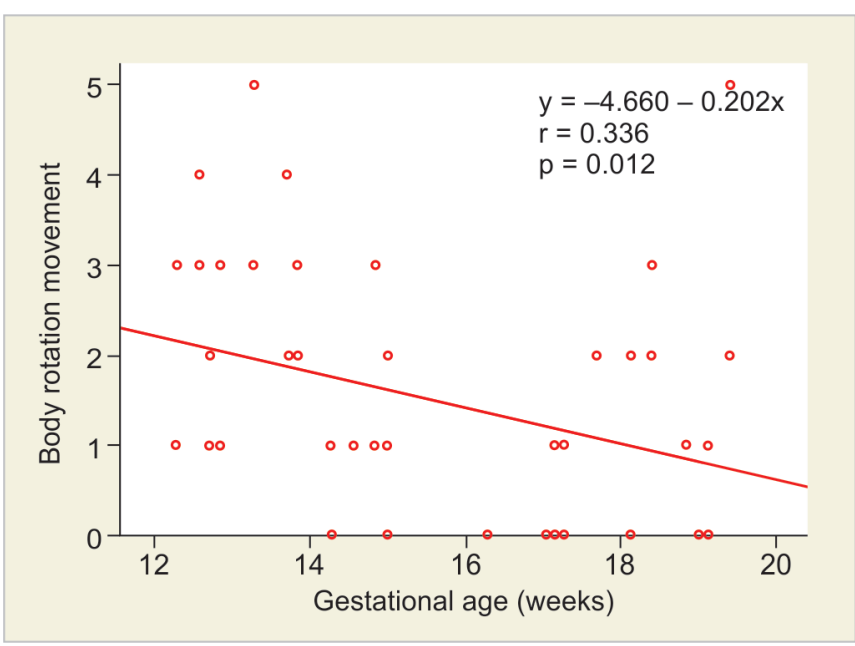

Graph 4: The frequency of twin body rotation movement by gestational age

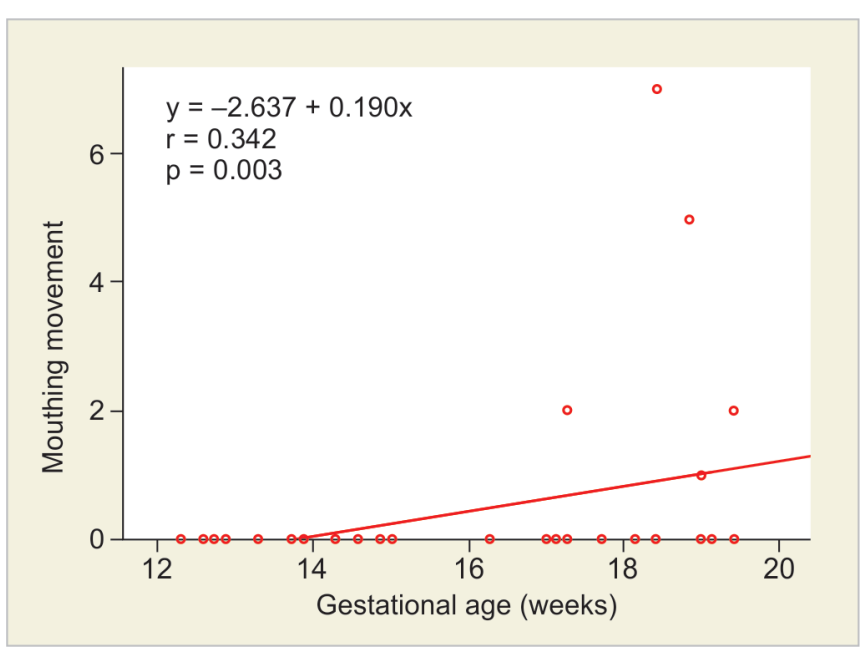

Graph 6: The frequency of twin mouthing movement by gestational age

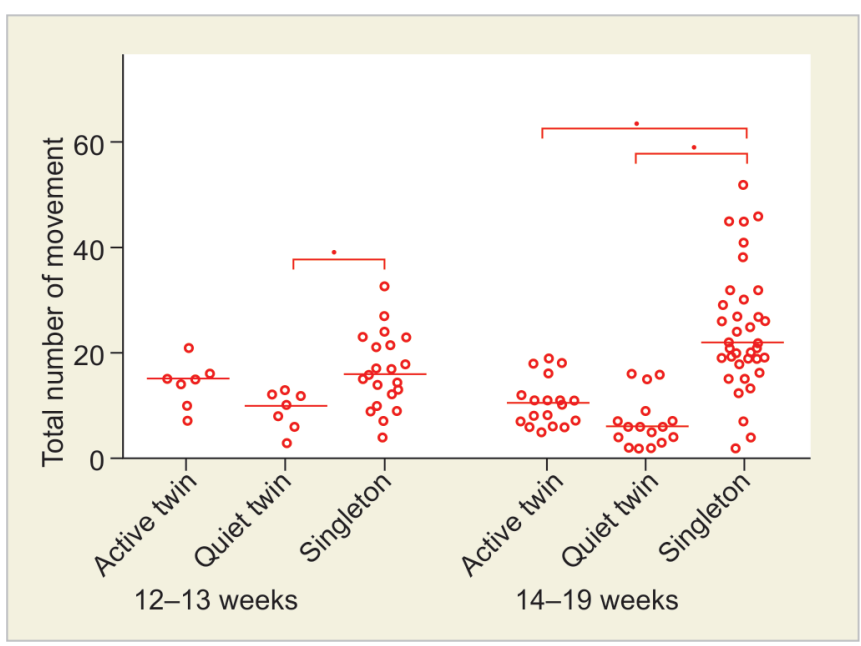

Graph 7: Comparison of the total number of fetal movements among singleton, active, and quiet twin fetuses at 12 to 13 and 14 to 19 weeks of gestation

a result, the frequencies of arm and general movements gradually increased from 8 to 19 weeks, whereas those of leg movement and head anteflexion showed similar 
incidences. The frequency of head retroflexion showed a decline after 12 weeks of gestation. Importantly, these authors stated that the hand-to-face movement could not be depicted because of the 2D character of images. In the present study, frequencies of hand-to-face and leg movements were significantly increased with advancing gestation, whereas the frequencies of general movements significantly decreased at 12 to 19 weeks in singleton fetuses. The frequencies of the other fetal movements did not change during this period. With respect to the major limitation of conventional 2D sonographic assessment, it has been pointed out that fetal movements outside the scanning plane cannot be displayed on the monitor screen because of the 2D character of real-time scanning. ${ }^{7,8}$ Therefore, the differences in the frequencies of fetal movements with advancing gestation before 20 weeks of gestation between 2D and 4D ultrasound assessments could be explained by the above-mentioned points. The $4 \mathrm{D}$ ultrasound is superior to 2D sonography for the assessment of fetal movements because of its three-dimensional imaging capabilities. ${ }^{7}$

As we previously reported, at 12 to 13 weeks, arm movement in twin fetuses was the only movement with a frequency significantly lower than in singleton fetuses; however, at 14 to 19 weeks, all eight movements in twin fetuses exhibited a frequency significantly lower than in singleton fetuses. ${ }^{5}$ It was suggested that the crowding of twin fetuses with gestation limits space, and that this has a marked impact on twin compared with singleton fetuses, even in the initial half of pregnancy. In the present study, frequencies of body rotation and general movements in twin fetuses significantly decreased with advancing gestation, at 12 to 19 weeks of gestation, whereas there were no significant differences in any other movements except for mouthing movement (weak increase during this period). The increase or decrease in the frequency of each fetal movement at 12 to 19 weeks between singleton and twin fetuses was mostly different. These differences may be due to the limited space and crowding in the womb of twin fetuses during the first half of pregnancy. As the restriction of fetal movements in twin pregnancies before 20 weeks of gestation may affect fetal growth and development or maturation before and after birth, further detailed studies involving a larger sample size are needed to investigate this hypothesis.

Mulder et $\mathrm{al}^{9}$ reported that twins showed a decrease in general movements with advancing gestation like singletons, but $72.5 \%$ of general movement values of twins were below the 50th percentile of the normal range for singletons. In the present study, the degree of decreasing frequency of general movements in twin fetuses was also steeper than that in singleton fetuses. These results may be also due to the limited space and crowding in the womb of twin fetuses during the first half of pregnancy.

Degani et $\mathrm{al}^{3}$ reported that the total counts of fetal movements (general body, isolated head, isolated arm, and isolated leg movements) between the active twins and their cotwins assessed by $4 \mathrm{D}$ ultrasound were significantly different between 11 and 14 weeks of gestation. Mulder et $\mathrm{al}^{9}$ noticed that twin fetuses were less active than singletons using 2D sonography throughout pregnancy. Piontelli et $\mathrm{al}^{10}$ also demonstrated that one twin was found to be "dominant" in the sense of being more active using 2D sonography at 10 to 22 weeks of gestation. In the present study, there was no significant difference in the total counts of fetal movements between the active twins and their cotwins at 12 to 13 weeks of gestation, although the total count of fetal movements in singleton fetuses was significantly higher than that in quiet twins. There was also no significant difference in total counts of fetal movements between active and quiet twins at 14 to 19 weeks of gestation. The discrepancy in the total counts of fetal movements between Degani et $\mathrm{al}^{3}$ study and the present study is currently unknown. One possible explanation is the difference in the number of fetal movements assessed between the studies (Degani et al study, 4 fetal movements; and our study, 8 fetal movements). Another possible explanation is the difference in the examination time (Degani et al study, 10 minutes; and our study, 15 minutes). Further studies with larger populations are needed to clarify the differences in motor activity between twin pairs.

\section{REFERENCES}

1. Kurjak A, Andonotopo W, Hafner T, Kadic AS, Stanojevic M, Azumendi G, Ahmed B, Carrera JM, Troyano JM. Normal standards for fetal neurobehavioral developments-longitudinal quantification by four-dimensional sonography. J Perinat Med 2006;34(1):56-65.

2. Sajapala S, AboEllail MAM, Kanenishi K, Mori N, Marumo G, Hata T. 4D ultrasound study of fetal movement early in the second trimester of pregnancy. J Perinat Med 2017 Aug;45(6): 737-743.

3. Degani S, Leibovitz Z, Shapiro I, Ohel G. Twins' temperament: early prenatal sonographic assessment and postnatal correlation. J Perinatol 2009 May;29(5):337-342.

4. Kurjak A, Talic A, Stanojevic M, Honemeyer U, Serra B, Prats P, Di Renzo GC. The study of fetal neurobehavior in twins in all three trimesters of pregnancy. J Matern Fetal Neonatal Med 2013 Aug;26(12):1186-1195.

5. AboEllail MAM, Kanenishi K, Mori N, Noguchi J, Marumo G, Hata T. Ultrasound study of fetal movements in singleton and twin pregnancies at 12-19 weeks. J Perinat Med 2017 Sep.

6. de Vries JIP, Visser GHA, Prechtl HFR. The emergence of fetal behaviour. II. Quantitative aspects. Early Hum Dev 1985 Nov;12(2):99-120. 
7. Kuno A, Akiyama M, Yamashiro C, Tanaka H, Yanagihara T, Hata T. Three-dimensional sonographic assessment of fetal behavior in the early second trimester of pregnancy. J Ultrasound Med 2001 Dec;20(12):1271-1275.

8. Hata T, Dai SY, Marumo G. Ultrasound for evaluation of fetal neurobehavioural development: from 2-D to 4-D ultrasound. Inf Child Dev 2010 Jan-Feb;19(1):99-118.
9. Mulder EJH, Derks JB, de Laat MWM, Visser GHA. Fetal behavior in normal dichorionic twin pregnancy. Early Hum Dev 2012 Mar;88(3):129-134.

10. Piontelli A, Bocconi L, Boschetto C, Kustermann A, Nicolini U. Differences and similarities in the intra-uterine behaviour of monozygotic and dizygotic twins. Twin Res 1999 Dec;2(4):264-273. 